Kragujevac Journal of Mathematics

Volume 42(4) (2018), Pages 527-532.

\title{
HORADAM SEQUENCE THROUGH RECURRENT DETERMINANTS OF TRIDIAGONAL MATRICES
}

\author{
TARAS GOY ${ }^{1}$
}

\begin{abstract}
Applying the apparatus of triangular matrices, we proved new recurrent formulas for Horadam numbers with even (odd) subscripts through determinants of tridiagonal matrix.
\end{abstract}

\section{Paradeterminant of Triangular Matrix}

In this section, we provide basic notions and results about paradeterminants of triangular matrices that will be used for the proving the main results of the paper.

Definition 1.1. [20] A triangular number table

$$
A_{n}=\left(\begin{array}{ccccc}
a_{11} & & & & \\
a_{21} & a_{22} & & & \\
\vdots & \vdots & \ddots & & \\
a_{n-1,1} & a_{n-1,2} & \cdots & a_{n-1, n-1} & \\
a_{n 1} & a_{n 2} & \cdots & a_{n, n-1} & a_{n n}
\end{array}\right)
$$

is called a triangular matrix, and the number $n$ is called its order.

Note that a matrix thus defined is not a matrix in the standard sense, because it is a triangular, rather than a rectangular, table of numbers.

The functions of triangular matrices are widely used in algebra, combinatorics, number theory, theory of ordinary differential equations, and other branches of mathematics (see [4,14,18-21] for more details and examples).

Key words and phrases. Horadam sequence, triangular matrix, tridiagonal matrix, paradeterminant, Fibonacci sequence.

2010 Mathematics Subject Classification. Primary: 11B39. Secondary: 11C20.

Received: July 23, 2016.

Accepted: May 23, 2017. 
Definition 1.2. [20] The paradeterminant of the triangular matrix (1.1), denote by $\operatorname{ddet}\left(A_{n}\right)$, is the number

$$
\operatorname{ddet}\left(A_{n}\right)=\sum_{r=1}^{n} \sum_{p_{1}+\cdots+p_{r}=n}(-1)^{n-r} \prod_{s=1}^{r}\left\{a_{p_{1}+\cdots+p_{s}, p_{1}+\cdots+p_{s-1}+1}\right\},
$$

where the summation is over the set of positive integer solutions of the equality $p_{1}+\cdots+p_{r}=n$, and $\left\{a_{i j}\right\}=a_{i j} \cdot a_{i, j+1} \cdots a_{i i}$.

For example,

$$
\begin{aligned}
\operatorname{ddet}\left(A_{4}\right)= & -a_{41} a_{42} a_{43} a_{44}+a_{31} a_{32} a_{33} a_{44}+a_{11} a_{42} a_{43} a_{44}+a_{21} a_{22} a_{43} a_{44} \\
& -a_{21} a_{22} a_{33} a_{44}-a_{11} a_{32} a_{33} a_{44}-a_{11} a_{22} a_{43} a_{44}+a_{11} a_{22} a_{33} a_{44} .
\end{aligned}
$$

The following formula (decomposition of a paradeterminant by elements of the last row) holds [20]:

$$
\operatorname{ddet}\left(A_{n}\right)=\sum_{s=1}^{n}(-1)^{n-s}\left\{a_{n s}\right\} \operatorname{ddet}\left(A_{s-1}\right)
$$

For example,

$$
\begin{aligned}
\operatorname{ddet}\left(A_{4}\right)= & -a_{41} a_{42} a_{43} a_{44} \operatorname{ddet}\left(A_{0}\right)+a_{42} a_{43} a_{44} \operatorname{ddet}\left(A_{1}\right) \\
& -a_{43} a_{44} \operatorname{ddet}\left(A_{2}\right)+a_{44} \operatorname{ddet}\left(A_{3}\right),
\end{aligned}
$$

where, by definition, $\operatorname{ddet}\left(A_{0}\right)=1$.

R. Zatorsky and I. Lishchynskyy [22] established the relation between the paradeterminants and the lower Hessenberg determinants by formula

$$
\operatorname{ddet}\left(A_{n}\right)=\left|\begin{array}{cccccc}
\left\{a_{11}\right\} & 1 & 0 & \ldots & 0 & 0 \\
\left\{a_{21}\right\} & \left\{a_{22}\right\} & 1 & \ldots & 0 & 0 \\
\left\{a_{31}\right\} & \left\{a_{32}\right\} & \left\{a_{33}\right\} & \ldots & 0 & 0 \\
\vdots & \vdots & \vdots & \ddots & \vdots & \vdots \\
\left\{a_{n-1,1}\right\} & \left\{a_{n-1,2}\right\} & \left\{a_{n-1,3}\right\} & \ldots & \left\{a_{n-1, n-1}\right\} & 1 \\
\left\{a_{n 1}\right\} & \left\{a_{n 2}\right\} & \left\{a_{n 3}\right\} & \ldots & \left\{a_{n, n-1}\right\} & \left\{a_{n n}\right\}
\end{array}\right| .
$$

\section{The Relation Between Horadam Numbers with Even (Odd) Subscripts AND PARAdETERminants}

For $n \geq 0$, the second order linear recurrence sequence $h_{n}=h_{n}(a, b ; p, q)$ is defined by

$$
h_{0}=a, \quad h_{1}=b, \quad h_{n+2}=p h_{n+1}-q h_{n},
$$

where $a, b, p, q$ are integers $(q \neq 0)$, was introduce by A. F. Horadam. The properties of this sequence were discussed in detail in $[1,2,5-7,13,15,16]$.

Sequence (2.1) generalized many well-known number sequences. Examples of such sequences are the Fibonacci, Lucas, Pell, Jacobsthal, Jacobsthal-Lucas, Pell-Lucas sequences, and some others sequences. 
Proposition 2.1. For $n \geq 1$, the following formula holds:

$$
h_{2 n-2}=\operatorname{ddet}\left(\begin{array}{cccccccc}
a & & & & & & \\
\frac{p}{q} \frac{h_{1}}{1} & -q & & & & & \\
0 & \frac{p}{q} \frac{h_{3}}{h_{0}} & -q & & & & \\
\vdots & \vdots & \vdots & \ddots & & & \\
0 & 0 & 0 & \ldots & -q & & \\
0 & 0 & 0 & \ldots & \frac{p}{q} \frac{h_{2 n-5}}{h_{2 n-8}} & -q & \\
0 & 0 & 0 & \ldots & 0 & \frac{p}{q} \frac{h_{2 n-3}}{h_{2 n-6}} & -q
\end{array}\right) \text {. }
$$

For example,

$$
\begin{aligned}
h_{0} & =a, \\
h_{2} & =\operatorname{ddet}\left(\begin{array}{cc}
a & \\
\frac{p}{q} \frac{h_{1}}{1} & -q
\end{array}\right) \\
& =-a q+b p, \\
h_{4} & =\operatorname{ddet}\left(\begin{array}{ccc}
a & \\
\frac{p}{q} \frac{h_{1}}{1} & -q & \\
0 & \frac{p}{q} \frac{h_{3}}{h_{0}} & -q
\end{array}\right) \\
& =a q^{2}-b p q+p\left(b p^{2}-a p q-b q\right) \\
& =a q^{2}-2 b p q+b p^{3}-a p^{2} q,
\end{aligned}
$$

and so on.

Proof. Expanding the paradeterminant (2.2) by elements of the last raw (see (1.2)), we have

$$
\begin{aligned}
h_{2 n-2} & =(-q) h_{2 n-4}-\frac{p}{q}(-q) \frac{h_{2 n-3}}{h_{2 n-6}} h_{2 n-6} \\
& =p h_{2 n-3}-q h_{2 n-4} .
\end{aligned}
$$

Thus, we obtained the recurrent relation (2.1).

Proposition 2.2. For $n \geq 1$, the following formula holds:

$$
h_{2 n-1}=\operatorname{ddet}\left(\begin{array}{cccccccc}
b & & & & & & \\
\frac{p}{q} \frac{h_{2}}{1} & -q & & & & & \\
0 & \frac{p}{q} \frac{h_{4}}{h_{1}} & -q & & & & \\
\vdots & \vdots & \vdots & \ddots & & & \\
0 & 0 & 0 & \ldots & -q & & \\
0 & 0 & 0 & \ldots & \frac{p}{q} \frac{h_{2 n-4}}{h_{2 n-7}} & -q & \\
0 & 0 & 0 & \ldots & 0 & \frac{p}{q} \frac{h_{2 n-2}}{h_{2 n-5}} & -q
\end{array}\right) \text {. }
$$


For example,

$$
\begin{aligned}
& h_{1}=b \text {, } \\
& h_{3}=\operatorname{ddet}\left(\begin{array}{cc}
b & \\
\frac{p}{q} \frac{h_{2}}{1} & -q
\end{array}\right) \\
& =-b q+p(b p-a q) \\
& =-b q+b p^{2}-a p q \text {, } \\
& h_{5}=\operatorname{ddet}\left(\begin{array}{ccc}
b & & \\
\frac{p}{q} \frac{h_{2}}{1} & -q & \\
0 & \underline{p} \frac{h_{4}}{h_{1}} & -q
\end{array}\right) \\
& =b q^{2}-p q(b p-a q)+p\left(a q^{2}-2 b p q+b p^{3}-a p^{2} q\right) \\
& =b q^{2}-3 b p^{2} q+2 a p q^{2}+b p^{4}-a p^{3} q \text {, }
\end{aligned}
$$

and so on.

Proof. The proof is similar to the Proposition 2.1. Indeed, using (1.2), we have

$$
\begin{aligned}
h_{2 n-1} & =(-q) h_{2 n-3}-\frac{p}{q}(-q) \frac{h_{2 n-2}}{h_{2 n-5}} h_{2 n-5} \\
& =p h_{2 n-2}-q h_{2 n-3} .
\end{aligned}
$$

\section{Main Results}

In this section, we prove new recurrent formulas expressing Horadam numbers $h_{n}$ with even (odd) subscripts through the determinants of tridiagonal matrix. As a consequence we obtain the corresponding formulas for Fibonacci numbers.

Theorem 3.1. For $n \geq 1$, the following formulas are hold:

$$
h_{2 n-2}=\frac{1}{\prod_{i=0}^{n-3} h_{2 i}} \cdot\left|\begin{array}{cccccccc}
a & 1 & 0 & 0 & \cdots & 0 & 0 & 0 \\
-p h_{1} & -q & 1 & 0 & \cdots & 0 & 0 & 0 \\
0 & -p h_{3} & -q h_{0} & h_{0} & \cdots & 0 & 0 & 0 \\
\vdots & \vdots & \vdots & \vdots & \ddots & \vdots & \vdots & \vdots \\
0 & 0 & 0 & 0 & \cdots & -p h_{2 n-5} & -q h_{2 n-8} & h_{2 n-8} \\
0 & 0 & 0 & 0 & \cdots & 0 & -p h_{2 n-3} & -q h_{2 n-6}
\end{array}\right|,
$$

and

$$
h_{2 n-1}=\frac{1}{\prod_{i=0}^{n-3} h_{2 i+1}} \cdot\left|\begin{array}{cccccccc}
b & 1 & 0 & 0 & \cdots & 0 & 0 & 0 \\
-p h_{2} & -q & 1 & 0 & \cdots & 0 & 0 & 0 \\
0 & -p h_{4} & -q h_{1} & h_{1} & \cdots & 0 & 0 & 0 \\
\vdots & \vdots & \vdots & \vdots & \ddots & \vdots & \vdots & \vdots \\
0 & 0 & 0 & 0 & \cdots & -p h_{2 n-4} & -q h_{2 n-7} & h_{2 n-7} \\
0 & 0 & 0 & 0 & \cdots & 0 & -p h_{2 n-2} & -q h_{2 n-5}
\end{array}\right|
$$


Proof. We prove the formula (3.1). Using (1.3), from (2.2) we have

$$
h_{2 n-2}=\left|\begin{array}{cccccccc}
a & 1 & 0 & 0 & \cdots & 0 & 0 & 0 \\
-p \frac{h_{1}}{1} & -q & 1 & 0 & \cdots & 0 & 0 & 0 \\
0 & -p \frac{h_{3}}{h_{0}} & -q & 1 & \cdots & 0 & 0 & 0 \\
\vdots & \vdots & \vdots & \vdots & \ddots & \vdots & \vdots & \vdots \\
0 & 0 & 0 & 0 & \cdots & -p \frac{h_{2 n-5}}{h_{2 n-8}} & -q & 1 \\
0 & 0 & 0 & 0 & \cdots & 0 & -p \frac{h_{2 n-3}}{h_{2 n-6}} & -q
\end{array}\right| .
$$

From this, after obvious transformations we get (3.1).

Formula (3.2) follows from (1.3) and (2.3).

Corollary 3.1. From (3.1), (3.2), for special choices of $a, b, p, q$ the following formulas can be obtained:

- Fibonacci numbers $F_{n}=h_{n}(1,1 ; 1,-1)$ (beginning at $\left.F_{1}=1\right)$ with even subscripts:

$$
F_{2 n}=\frac{1}{F_{2} F_{4} \cdots F_{2 n-4}}\left|\begin{array}{cccccccc}
1 & 1 & 0 & 0 & \cdots & 0 & 0 & 0 \\
-F_{3} & 1 & 1 & 0 & \cdots & 0 & 0 & 0 \\
0 & -F_{5} & F_{2} & F_{2} & \cdots & 0 & 0 & 0 \\
\vdots & \vdots & \vdots & \vdots & \ddots & \vdots & \vdots & \vdots \\
0 & 0 & 0 & 0 & \cdots & -F_{2 n-3} & F_{2 n-6} & F_{2 n-6} \\
0 & 0 & 0 & 0 & \cdots & 0 & -F_{2 n-1} & F_{2 n-4}
\end{array}\right| ;
$$

- Fibonacci numbers (beginning at $F_{1}=1$ ) with odd subscripts:

$$
F_{2 n-1}=\frac{1}{F_{1} F_{3} \cdots F_{2 n-5}}\left|\begin{array}{cccccccc}
1 & 1 & 0 & 0 & \cdots & 0 & 0 & 0 \\
-F_{2} & 1 & 1 & 0 & \cdots & 0 & 0 & 0 \\
0 & -F_{4} & F_{1} & F_{1} & \cdots & 0 & 0 & 0 \\
\vdots & \vdots & \vdots & \vdots & \ddots & \vdots & \vdots & \vdots \\
0 & 0 & 0 & 0 & \cdots & -F_{2 n-4} & F_{2 n-7} & F_{2 n-7} \\
0 & 0 & 0 & 0 & \cdots & 0 & -F_{2 n-2} & F_{2 n-5}
\end{array}\right| .
$$

By choosing other suitable values on $a, b, p$ and $q$, one can also obtain the Lucas, Pell, Jacobsthal, Jacobsthal-Lucas and Pell-Lucas numbers or polynomials in term of recurrent determinants of tridiagonal matrix.

Note that determinants of matrices, elements of which are classical or generalized Fibonacci and Lucas numbers, in particular, studied in $[3,8-12,17]$.

Acknowledgements. The author would like to thank the anonymous referee for his/her valuable comments and suggestions for improving the original version of this article. The author also is grateful to professor Roman Zatorsky, Faculty of Mathematics and Computer Sciences Vasyl Stefanyk Precarpathian National University (Ukraine), for constant attention to this work and for useful discussions. 


\section{REFERENCES}

[1] Z. Akyuz and S. Halici, On some combinatorial identities involving the terms of generalized Fibonacci and Lucas sequences, Hacet. J. Math. Stat. 42(4) (2013), 431-435.

[2] G. Cerda-Morales, On generalized Fibonacci and Lucas numbers by matrix method, Hacet. J. Math. Stat. 42(2) (2013), 173-179.

[3] H. Civciv, A note on the determinant of five-diagonal matrices with Fibonacci numbers, Int. J. Contemp. Math. Sci. 3(9) (2008), 419-424.

[4] T. P. Goy and R. A. Zatorsky, Using triangular matrices for construction of ordinary differential equations for the known fundamental system of solutions, NaUKMA Sci. Notes. Ser. Phys. Math. 165 (2015), 3-6.

[5] A. F. Horadam, Basic properties of a certain generalized sequence of numbers, Fibonacci Quart. 3(3) (1965), 161-176.

[6] A. F. Horadam, Generating function of a certain generalized sequence of numbers, Duke Math. J. 32(3) (1965), 437-446.

[7] A. F. Horadam and A. G. Shannon, Generalization of identities of Catalan and others, Port. Math. 44(2) (1987), 137-148.

[8] A. İpek, On the determinants of pentadiagonal matrices with the classical Fibonacci, generalized Fibonacci and Lucas numbers, Eurasian Math. J. 2(2) (2011), 60-74.

[9] A. İpek and K. Arı, On Hessenberg and pentadiagonal determinants related with Fibonacci and Fibonacci-like numbers, Appl. Math. Comput. 229 (2014), 433-439.

[10] T. Koshy, Fibonacci and Lucas Numbers with Applications, John Wiley \& Sons, 2001.

[11] H. Kwong, Two determinants with Fibonacci and Lucas entries, Appl. Math. Comput. 194(2) (2007), 568-571.

[12] A. J. Macfarlane, Use of determinants to present identities involving Fibonacci and related numbers, Fibonacci Quart. 48(1) (2010), 68-76.

[13] A. G. Shannon, Fibonacci numbers and Diophantine quadruples: generalization of result of Morgado and Horadam, Port. Math. 45(2) (1988), 165-169.

[14] V. E. Tarakanov and R. A. Zatorskii, A relationship between determinants and permanents, Math. Notes 85(1) (2009), 267-273.

[15] G. Udrea, A note on the sequence $\left(W_{n}\right)_{n \geq 0}$ of A.F. Horadam, Port. Math. 53(2) (1996), 143-155.

[16] Y. Yazlik and N. Taskara, A note generalized $k$-Horadam sequence, Comput. Math. Appl. 63 (2012), 36-41.

[17] F. Yilmaz and D. Bozkurt, On the Fibonacci and Lucas numbers, their sums and permanents of one type of Hessenberg matrices, Hacet. J. Math. Stat. 43(6) (2014), 1001-1006.

[18] R. A. Zatorsky, Determinants of triangular matrices and trajectories on Ferre diagrams, Math. Notes 72(5) (2002), 768-783.

[19] R. Zatorsky, Introduction to the theory of triangular matrices (tables), in: I. I. Kyrchey (Ed.), Advances in Linear Algebra Research, Nova Science Publishers, New York, 2015, pp. 185-238.

[20] R. A. Zatorsky, Theory of paradeterminants and its applications, Algebra Discrete Math. 1 (2007), 108-137.

[21] R. Zatorsky and T. Goy, Parapermanents of triangular matrices and some general theorems on number sequences, J. Integer Seq. 19 (2016), Article 16.2.2, 22 pages.

[22] R. A. Zatorsky and I. I. Lishchynskyy, On connection between determinants and paradeterminants, Mat. Stud. 25 (2006), 97-102.

${ }^{1}$ Faculty of Mathematics and Computer Sciences, Vasyl Stefanyk Precarpathian National University, IVANO-FRANKIVSK, UKRAINE

Email address: tarasgoy@yahoo.com 